\section{Acoustic interference in paired-associate learning as a function of hearing ability ${ }^{1}$}

DORIS V. ALLEN, School of Medicine, Wayne State University, Detroit, Mich. 48201

Qualitative differences in coding verbal materials were postulated between normaland impaired-hearing children. Acoustic similarity was used in two paired-associate lists differing in manner of pairings. The regression of performance upon hearing loss was significant for consistent pairings but not for inconsistent pairings. The results supported the hypothesized qualitative differences.

The basic hypothesis tested was that qualitative differences in processing of verbal materials result from a congenital hearing impairment. It was postulated that normal-hearing Ss employ implicit acoustic features of printed materials, while hearing-handicapped Ss encode the material by using visual aspects.

A number of studies comparing performance of normal-hearing and impaired-hearing groups lend support to the hypothesized qualitative differences. For example, Conrad \& Rush (1965) found that normal-hearing adults made acoustic errors (e.g., substituting $B$ for $C$ in recalling sequences of letters) in a short-term memory task; they further found that deaf adults also made consistent errors, but along a dimension that could not be identified. Odom \& Blanton (1967) studied phrase learning by deaf and normal-hearing children and concluded that the deaf processed word phrases differently. Two other studies demonstrated that deaf performance was not affected by the pronunciability ratings of items in contrast to the interference exhibited by difficult items upon normal-hearing performance. Youniss (1964) reported that the deaf were better than normals on training trials in a study of concept transfer, although the groups did not differ on the transfer phase of the experiment. He conjectured that the deaf Ss' "lack of verbal experience did not impede their using some other surrogate mechanism comparable to verbal mediating responses." Thus, it can be seen that a number of investigators have noted a qualitative difference in performance between normal-hearing and hearing-handicapped Ss.

One way of gaining insight into the cognitive processes involved in learning is to identify factors interfering with such learning. A study by Dallett (1966) provides such information. He explored the relative effects of interference on both acquisition and retention of paired-associate lists. Interference was introduced by manipulating acoustic similarity, using two lists, so that similarity existed either between lists or within lists. He further arranged his materials to form pairings that were either "consistent" or "not consistent," the designations referring to whether homophonous stimuli had rhyming responses (consistent pairings) or responses that did not rhyme (not consistent pairings). Using trials-to-criterion for his data and college students as Ss, he found that within-list similarity produced significant interference as compared to a control list and that pairings that were not consistent produced greater interference with acquisition than did the consistent pairings.

Dallett's finding that acoustic similarity interfered with learning reinforces the hypothesis that normal-hearing Ss use implicit aural attributes of words as cues for learning. This effect was used in this experiment to gain evidence relevant to the basic thesis that qualitative differences in thinking processes occur as a function of hearing ability. Simply stated, if hearing-handicapped Ss do not use the same acoustic cues, then interference will not occur and their performance will exceed that of normal-hearing Ss.

A study was designed using Dallett's model for consistent and inconsistent within-list pairings. Children operating at about the fourth-grade level were chosen as Ss since that represents the usual level of achievement for the congenitally deaf individual. No control list was used since the primary interest was in the relative amounts of interference obtained as a function of manner of pairings and hearing ability. It was expected that normal-hearing Ss would perform more poorly on the inconsistent than on the consistent list, while hearing-handicapped Ss would show no difference as a function of pairings of words, and that normal hearing performance on either list would be poorer than hearing-impaired performance. Thus, an interaction between hearing ability and pairings of words was predicted.

\section{MATERIALS}

Eight pairs of rhyming words were used. The words were spelled differently although sounding alike (e.g., DOOR-MORE), All words were monosyllabic, with either A or AA frequency ratings (Thorndike \& Lorge, 1944). The eight pairs were arbitrarily divided into two equal sets, one set becoming stimuli and the other responses. Stimulus-response pairings were then formed so as to be consistent in one list and inconsistent in the other, as defined by Dallett. For example, two rhyming pairs (DOOR-MORE and SIGH-LIE) were taken; the first set was assigned to the stimulus category and the other became responses. In the consistent list, rhyming stimuli had rhyming responses, i.e., DOOR was paired with SIGH and MORE with LIE, while, in the list with inconsistent pairings, the pairs were DOOR-SIGH and MORE-WHILE, the response LIE being paired with another stimulus. Each list thus contained identical stimulus and response words; only the way in which they were paired was varied. A practice list of four unrelated words was also used.

\section{SUBJECTS}

Three degrees of hearing ability were defined, using the average of the unaided pure-tone thresholds (PTA) for the better ear for $500,1,000$, and $2,000 \mathrm{~Hz}$. Ss with losses averaging 0-25 dB (ISO) formed one category termed "essentially normal." Losses of this magnitude are generally considered nonsignificant and such children are usually found in regular classrooms, perhaps with favorable seating being recommended. The next category included those with losses from 26 to $65 \mathrm{~dB}$; this category might be called "hard of hearing." The third category consisted of those with losses of $66 \mathrm{~dB}$ or greater and included severe and profound hearing impairments. All Ss had reading levels (RL) better than Grade 3.5 but not exceeding Grade 6, a range of ability generally found in a fourth-grade classroom. All losses were sensorineural and predated the age of the onset of speech. Table 1 summarizes the

Table 1

Descriptions of Groups Receiving the Two Experimental Lists

\begin{tabular}{|c|c|c|c|c|c|c|c|c|c|c|}
\hline \multirow[b]{3}{*}{ Group } & \multicolumn{5}{|c|}{ List $C$} & \multicolumn{5}{|c|}{ List I } \\
\hline & \multicolumn{2}{|c|}{$N$} & \multirow[b]{2}{*}{ Age } & \multirow[b]{2}{*}{ PTA } & \multirow[b]{2}{*}{ RL } & \multicolumn{2}{|c|}{$\mathrm{N}$} & \multirow[b]{2}{*}{ Age } & \multirow[b]{2}{*}{ PTA } & \multirow[b]{2}{*}{ RL } \\
\hline & $\mathbf{M}$ & $\mathbf{F}$ & & & & $\mathrm{M}$ & $\mathbf{F}$ & & & \\
\hline $0-25$ & 6 & 4 & 10.5 & 5.3 & 4.3 & 4 & 7 & 13.2 & 8.6 & 4.5 \\
\hline $26-65$ & 3 & 3 & 11.5 & 48.0 & 3.8 & 8 & 0 & 11.6 & 47.5 & 4.0 \\
\hline $66+$ & 6 & 6 & 13.5 & 93.4 & 4.5 & 5 & 7 & 11.8 & 86.1 & 3.5 \\
\hline
\end{tabular}


Table 2

Mean Performance by Four Categories of Hearing Ability on Two Lists

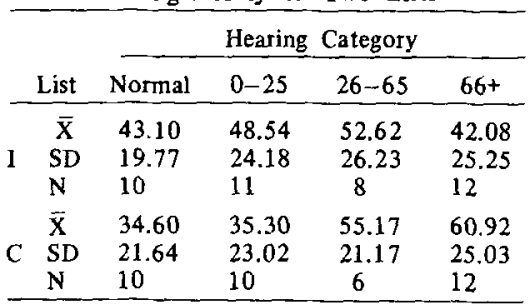

relevant data for the three groups of children receiving each list. As shown there, a total of $59 \mathrm{Ss}$ participated in the study, 28 learning List $C$ and 31 List I. Ss from each category were assigned alternately to a list as they appeared for testing.

\section{PROCEDURES}

All Ss were tested individually. Materials were presented visually, using a projector and a $16-\mathrm{mm}$ film strip. Responses were written in booklets provided. The list of unrelated word pairs was administered initially to insure comprehension of the task requirements. Alternating study and test trials were used until the $S$ reached one perfect trial on the practice list. Then one of the experimental lists was administered for a total of 12 sets of alternating study-test trials. Each $S$ received only one experimental list.

\section{RESULTS}

The mean numbers of correct responses for each list for each hearing category were determined. Table 2 summarizes these data and Fig. 1 illustrates the results. Included are data for small samples of normal-hearing Grade 4 Ss randomly selected from group data. These values are included primarily to provide an index of normal-hearing performance as a check upon the assumption that the group with $0-25 \mathrm{~dB}$ hearing loss was "essentially normal." The "normal" data were obtained for other purposes; group testing conditions without a practice list were used so the relative performances should be compared with caution. (These values have since been validated using individual testing and a practice list.)

If the normal data points are ignored in Fig. 1, the trend seems to be linear and positive for the consistent list and about zero for the inconsistent list across the remaining hearing categories. However, a $2 \times 3$ analysis of variance of the data showed no significant sources of variance. The List by Hearing Loss interaction $[F(2,53)=2.42]$ was the largest source of variance but would only be significant at the $10 \%$ level, thus not lending support to

Fig. 2. Learning curves for four hearing categories with List $\mathrm{C}$.

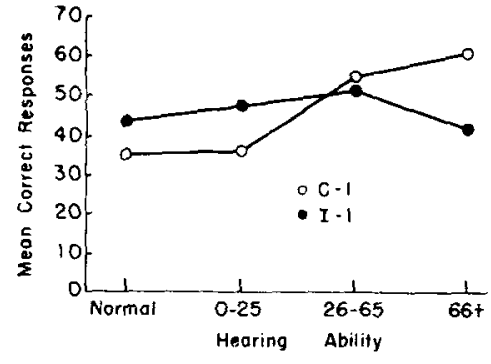

Fig. 1. Mean performance by hearing category on two experimental lists.

the trend. Small samples and large category widths, resulting in large within-cell variance, were undoubtedly factors in this. Using $t$ tests, the difference between the two lists for the group with $66+$ hearing loss was significant $[\mathrm{t}(22)=1.84, \mathrm{p}<.05]$, as was the difference between the $0-25$ and $66+$ groups on List C $[t(20)=2.48$, $p<.05]$. Following up the apparent trends illustrated in Fig. 1, regression coefficients for the two lists were obtained. The regression of performance on hearing loss for the inconsistent list was -.07 , which is not significantly different from zero, while the value for the consistent list was .24 , significant at the $5 \%$ level.

The observed trend to better performance with increasing hearing loss on the list with consistent pairings was thus supported statistically. As would be expected, correlation coefficients agreed with the regression coefficients; $r$ for PTA and performance on List I was - .10 and on List $C$ was .39. Again, only the latter value is significant at the .05 level. Since age and hearing loss tend to covary when groups are equated on reading level, corresponding correlations for age and performance were determined. These values were .51 and .18 for Lists $I$ and $C$, respectively. Only the former, for List $\mathrm{I}$, is significant. The lack of significance for List $C$ insures that age and hearing loss are not being confounded.

Learning curves for the two lists provide additional evidence for the hypothesis that hearing loss interacts with manner of pairings. Figure 2 shows learning curves for the four hearing categories for List C. Again, the normal Grade 4 samples are included, primarily for reference. As shown in this figure, the groups form two distinct clusters in rate of acquisition of the consistent list. The upper curves are for the two more seriously impaired hearing categories, $26-65$ and $66+\mathrm{dB}$ hearing loss, while the lower curves are for $0-25$ $\mathrm{dB}$ loss and normal groups. These data suggest that a moderate or severe hearing impairment facilitates learning the consistent list, or, conversely, that Ss with normal hearing or with only very mild hearing losses perform less efficiently on that list. The clear separation in rate of acquisition of List $\mathrm{C}$ as a function of hearing ability is in sharp contrast to the overlapping curves for List I, seen in Fig. 3 . These curves indicate that the groups did not differ appreciably in rate of learning the inconsistent list.

\section{DISCUSSION}

In terms of the specific hypotheses tested in this experiment, the results are the reverse of the predictions: (1) Normal-hearing children performed alike on the two lists, while Ss with severe hearing losses $(66+\mathrm{dB})$ performed significantly better with consistent pairings than with inconsistent pairings; and (2) normal-hearing performance was poorer than that of hearing-impaired Ss only on

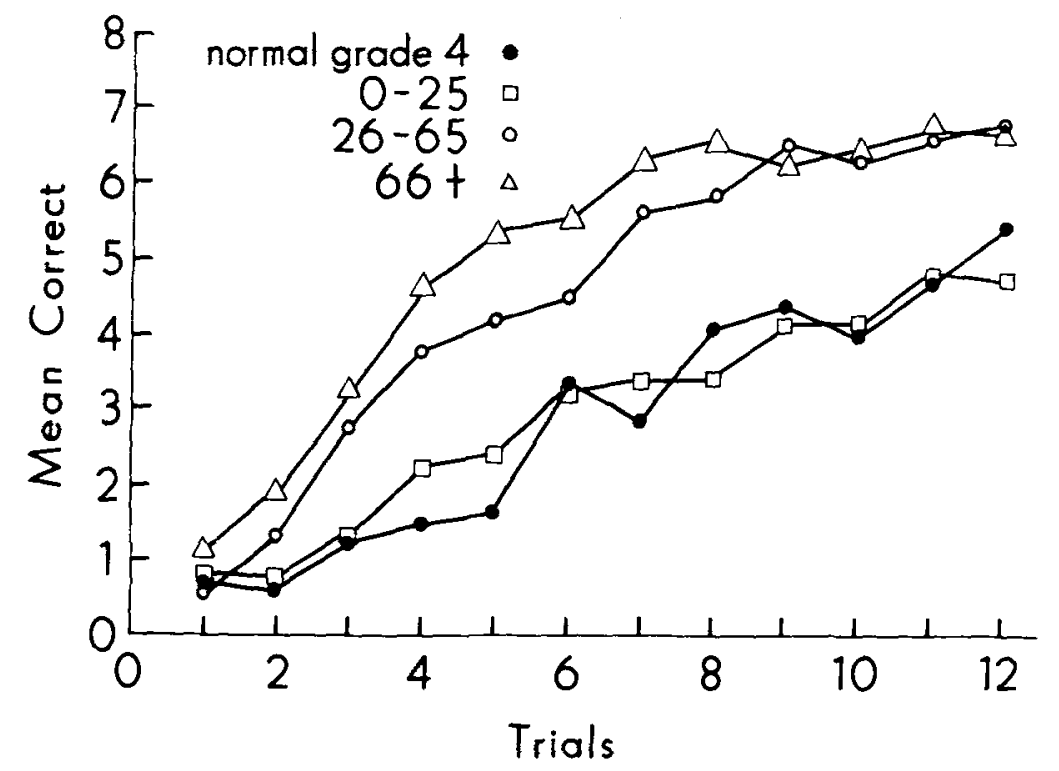


Fig. 3. Learning curves for four hearing categories with List 1 .

the consistent list. However, the predicted interaction between pairings and hearing was supported by the slopes of the functions relating performance to PTA, this being zero for the inconsistent list and significantly positjve for the consistent list.

Both normal-hearing Grade $4 \mathrm{Ss}$ in group testing and the $0.25 \mathrm{~dB}$ hearing-loss Ss in individual testing failed to perform differently on the two lists. Thus, the anticipated interference effect with the inconsistent list, predicted on the basis of Dallett's results, did not hold for this study. A number of procedural differences between Dallett's study and the present experiment may account for this discrepancy. Perhaps the most critical difference lies in the age groupings used, college students vs Grade 4 children. It may be that the results obtained by Dallett cannot be generalized to younger Ss. However, the fact that children with severe hearing losses performed differently suggests that this explanation is not sufficient. To find hearing-handicapped children performing better than normals on any verbal task is contrary to all previous evidence and indicates that something about the materials themselves produced the differential performance.

The most significant finding emerging from this study is the fact just mentioned, that a hearing loss seemed to insure a trend to better performance, at least on the consistent list. This result, coupled with a clear distinction in the rate of learning that same list by Ss with and without significant hearing losses, provides additional support for the basic hypothesis in this experiment.

It had been postulated that normal-hearing and hearing-impaired differ in the way they manipulate verbal material internally. Certainly, the data from the consistent list indicate that the greater the hearing loss, the less interference obtained from acoustically similar words. Why this same effect was not noted with List I is not fully understood. The same words were used in either list and interference from acoustic similarity was found with both sets of pairings by Dallett. Thus, reduction in interference was expected on both lists with hearing-handicapped children but was not obtained.

On the basis of these results, one may conjecture that the hearing-loss Ss use physical appearance of the printed words as a primary dimension for encoding in contrast to the normal-hearing Ss' use of the implicit acoustic characteristics of the materials. Thus, the hearing-handicapped were able to circumvent the source of interference. The better performance on

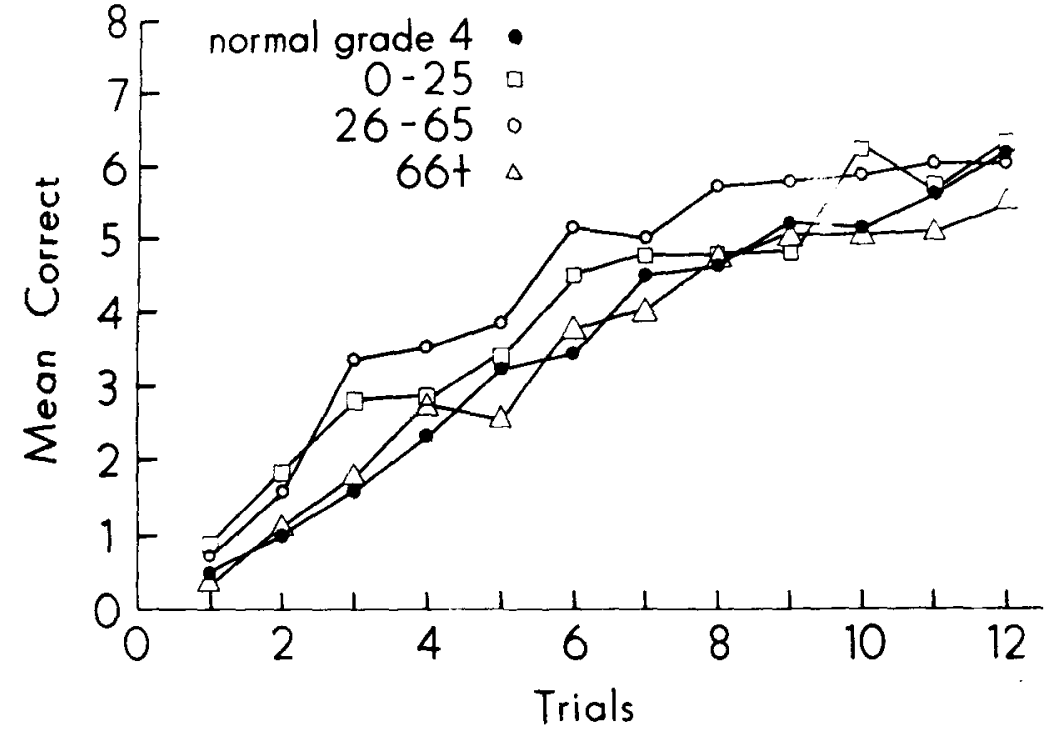

the consistent list by this group suggests that they were able to use the rhyming quality as a cue to aid learning. This facilitation would have to come from use of the auditory characteristics of the materials as a secondary attribute for encoding, since primary attention to this dimension would result in response generalization and interference. Thus, it may be that, to the normal-hearing child, a word is "thought of" first as a pattern of sounds and then as a set of orthographic symbols. In contrast, to a person with a congenital hearing loss, a word is first responded to as a set of letters and then, secondarily, as a pattern of phonemes, with the latter representing a learned response acquited through auditory training. Ultimately, of course, both kinds of Ss "think of" a word in terms of its meaning.

\section{REFERENCES}

BLANTON, R. L., \& NUNNALLY, J. C Retention of trigrams by deaf and hearing subjects as a function of pronunciability. Journal of Verbal Learning \& Verbal Behavior $1967,6,428-431$.
BLANTON, R. L., \& ODOM, P. B. Some possible interference and facilitation effects of pronunciability. Journal of Verbal Learning \& Verbal Behavior, 1968, 7, 844-846.

CONRAD, R., \& RUSH, M. L. On the nature of short-term memory encoding by the deaf. Journal of Speech \& Hearing Disorders, 1965 , 30, 336-343.

DALLETT, K. M. The effects of within-list and between-list acoustic similarity on the leaming and retention of paired associates. Joumal of Experimental Psychology, 1966, 72, 667-677.

ODOM, P. B., \& BLANTON, R. L. Phrase-learning in deaf and hearing subjects. Journal of Speech \& Hearing Research, 1967, 10,600-605.

THORNDIKE, E. L., \& LORGE, I. The teacher's word book of 30,000 words. New York: Bureau of Publications, Teachers College, Columbia University, 1944.

YOUNISS, J. Concept transfer as a function of shifts, age, and deafness. Child Development, $1964,35,695-700$.

\section{NOTE}

1. The work reported herein was performed pursuant to Grant No. OEG-0-8-070837-1858 from the U.S. Office of Education, Department of Health, Education, and Welfare.

\section{Short-term memory encoding strategies of the deaf!}

\section{JOHN L. LOCKE, ${ }^{2}$ University of Illinois, Champaign, Ill. 61820}

Deaf Ss, using an $A B X$ procedure, evaluated nine consonant letters in terms of the subjective kinesthetic similarity of their dactylic representations. Ranked data were compared to short-term memory recall errors for the same stimuli in a similar group of deaf $S s$ (Conrad \& Rush, 1965). Findings suggest that deaf $S s$ do not encode orthographic stimuli with a dactylo-kinesthetic system exclusively, if at all.

A series of experiments (Conrad, 1962, 1963; Wickelgren, 1965a, b, c) has established that short-term memory (STM) errors in verbal learning are predictable on the basis of the phonetic configuration of the stimulus items, regardless of whether the mode of presentation is auditory or visual. Apparently, Ss somehow convert a strictly visual language stimulus, such as an 